\title{
Multivariate normal approximation of the maximum likelihood estimator via the delta method
}

\author{
Andreas Anastasiou ${ }^{a}$ \\ and \\ Robert E. Gaunt ${ }^{\mathrm{b}}$ \\ ${ }^{\mathrm{a}}$ The London School of Economics and Political Science \\ $\mathrm{b}$ The University of Manchester
}

\begin{abstract}
We use the delta method and Stein's method to derive, under regularity conditions, explicit upper bounds for the distributional distance between the distribution of the maximum likelihood estimator (MLE) of a $d$-dimensional parameter and its asymptotic multivariate normal distribution. Our bounds apply in situations in which the MLE can be written as a function of a sum of i.i.d. $t$-dimensional random vectors. We apply our general bound to establish a bound for the multivariate normal approximation of the MLE of the normal distribution with unknown mean and variance.
\end{abstract}

\section{Introduction}

The asymptotic normality of maximum likelihood estimators (MLEs), under regularity conditions, is one of the most well-known and fundamental results in mathematical statistics. In a very recent paper, [1] obtained explicit upper bounds on the distributional distance between the distribution of the MLE of a $d$-dimensional parameter and its asymptotic multivariate normal distribution. In the present paper, we use a different approach to derive such bounds in the special situation where the MLE can be written as a function of a sum of i.i.d. $t$-dimensional random vectors. In this setting, our bounds improve on (or are at least as good as) those of [1] in terms of sharpness, simplicity and strength of distributional distance. The process used to derive our bounds consists a combination of Stein's method and the multivariate delta method. It is a natural generalisation of the approach used by [2] to obtain improved bounds - only in cases where the MLE can be expressed as a function of a sum of i.i.d. random variables - than those obtained by [3] for the asymptotic normality of the single parameter MLE.

MSC 2010 subject classifications: Primary 60F05, 62E17, 62F12

Keywords and phrases. Multi-parameter maximum likelihood estimation, multivariate normal distribution, Stein's method 
Let us now introduce the notation and setting of this paper. Let $\boldsymbol{\theta}=$ $\left(\theta_{1}, \ldots, \theta_{d}\right)$ denote the unknown parameter found in a statistical model. Let $\boldsymbol{\theta}_{\mathbf{0}}=\left(\theta_{0_{1}}, \ldots, \theta_{0_{d}}\right)$ be the true (still unknown) value and let $\boldsymbol{\Theta} \subset \mathbb{R}^{d}$ denote the parameter space. Suppose $\mathbf{X}=\left(\mathbf{X}_{1}, \ldots, \mathbf{X}_{n}\right)$ is a random sample of $n$ i.i.d. $t$-dimensional random vectors with joint probability density (or mass) function $f(\boldsymbol{x} \mid \boldsymbol{\theta})$, where $\boldsymbol{x}=\left(\boldsymbol{x}_{1}, \boldsymbol{x}_{2}, \ldots, \boldsymbol{x}_{n}\right)$. The likelihood function is $L(\boldsymbol{\theta} ; \boldsymbol{x})=f(\boldsymbol{x} \mid \boldsymbol{\theta})$, and its natural logarithm, called the log-likelihood function, is denoted by $l(\boldsymbol{\theta} ; \boldsymbol{x})$. We shall assume that the MLE $\hat{\boldsymbol{\theta}}_{\boldsymbol{n}}(\boldsymbol{X})$ of the parameter of interest $\boldsymbol{\theta}$ exists and is unique. It should, however, be noted that uniqueness and existence of the MLE can not be taken for granted; see, for example, [4] for an example of non-uniqueness. Assumptions that ensure existence and uniqueness of the MLE are given in [9].

In addition to assuming the existence and uniqueness of the MLE, we shall assume that the MLE takes the following form. Let $q: \Theta \rightarrow \mathbb{R}^{d}$ be such as all the entries of the function $q$ have continuous partial derivatives with respect to $\boldsymbol{\theta}$ and that

$$
q\left(\hat{\boldsymbol{\theta}}_{\boldsymbol{n}}(\boldsymbol{x})\right)=\frac{1}{n} \sum_{i=1}^{n} g\left(\boldsymbol{x}_{\boldsymbol{i}}\right)=\frac{1}{n} \sum_{i=1}^{n}\left(g_{1}\left(\boldsymbol{x}_{\boldsymbol{i}}\right), \ldots, g_{d}\left(\boldsymbol{x}_{\boldsymbol{i}}\right)\right)^{\top}
$$

for some $g: \mathbb{R}^{t} \rightarrow \mathbb{R}^{d}$. This setting is a natural generalisation of that of [2] to multiparameter MLEs. The representation (1.1) for the MLE allows us to approximate the MLE using a different approach to that given in [1], which results in a bound that improves on that obtained by [1]. As we shall see in Section 3, a simple and important example of an MLE of the form (1.1) is given by the normal distribution with unknown mean and variance:

$$
f(x \mid \boldsymbol{\theta})=\frac{1}{\sqrt{2 \pi \sigma^{2}}} \exp \left\{-\frac{1}{2 \sigma^{2}}(x-\mu)^{2}\right\}, \quad x \in \mathbb{R} .
$$

In this paper, we derive general bounds for the distributional distance between an MLE of the form (1.1) and its limiting multivariate normal distribution. The rest of the paper is organised as follows. In Section 2, we derive a general upper bound on the distributional distance between the distribution of the MLE of a $d$-dimensional parameter and the limiting multivariate normal distribution for situations in which (1.1) is satisfied. In Section 3, we apply our general bound to obtain a bound for the multivariate normal approximation of the MLE of the normal distribution with unknown mean and variance.

imsart-bjps ver. 2014/10/16 file: mle_arxiv.tex date: August 10, 2018 


\section{The general bound}

In this section, we give quantitative results in order to compliment the qualitative result for the multivariate normal approximation of the MLE as expressed below in Theorem 2.1. In the statement of this theorem, and the rest of the paper, $I\left(\boldsymbol{\theta}_{\mathbf{0}}\right)$ denotes the expected Fisher information matrix for one random vector, while $\left[I\left(\boldsymbol{\theta}_{\mathbf{0}}\right)\right]^{\frac{1}{2}}$ denotes the principal square root of $I\left(\boldsymbol{\theta}_{\mathbf{0}}\right)$. Also, $I_{d \times d}$ denotes the $d \times d$ identity matrix. To avoid confusion with the expected Fisher information matrix, the subscript will always be included in the notation for the $d \times d$ identity matrix. Following [6], the following assumptions are made in order for the asymptotic normality of the vector MLE to hold:

(R.C.1) The densities defined by any two different values of $\boldsymbol{\theta}$ are distinct.

(R.C.2) $\ell(\boldsymbol{\theta} ; \boldsymbol{x})$ is three times differentiable with respect to the unknown vector parameter, $\boldsymbol{\theta}$, and the third partial derivatives are continuous in $\boldsymbol{\theta}$.

(R.C.3) For any $\boldsymbol{\theta}_{\mathbf{0}} \in \boldsymbol{\Theta}$ and for $\mathbb{X}$ denoting the support of the data, there exists $\epsilon_{0}>0$ and functions $M_{r s t}(\boldsymbol{x})$ (they can depend on $\boldsymbol{\theta}_{\mathbf{0}}$ ), such that for $\boldsymbol{\theta}=\left(\theta_{1}, \theta_{2}, \ldots, \theta_{d}\right)$ and $r, s, t, j=1,2, \ldots, d$,

$$
\left|\frac{\partial^{3}}{\partial \theta_{r} \partial \theta_{s} \partial \theta_{t}} \ell(\boldsymbol{\theta} ; \boldsymbol{x})\right| \leq M_{r s t}(\boldsymbol{x}), \forall \boldsymbol{x} \in \mathbb{X},\left|\theta_{j}-\theta_{0, j}\right|<\epsilon_{0},
$$

with $\mathbb{E}\left[M_{r s t}(\boldsymbol{X})\right]<\infty$.

(R.C.4) For all $\boldsymbol{\theta} \in \Theta, \mathbb{E}_{\boldsymbol{\theta}}\left[\ell_{\boldsymbol{X}_{\boldsymbol{i}}}(\boldsymbol{\theta})\right]=0$.

(R.C.5) The expected Fisher information matrix for one random vector $I(\boldsymbol{\theta})$ is finite, symmetric and positive definite. For $r, s=1,2, \ldots, d$, its elements satisfy

$$
n[I(\boldsymbol{\theta})]_{r s}=\mathbb{E}\left[\frac{\partial}{\partial \theta_{r}} \ell(\boldsymbol{\theta} ; \boldsymbol{X}) \frac{\partial}{\partial \theta_{s}} \ell(\boldsymbol{\theta} ; \boldsymbol{X})\right]=\mathbb{E}\left[-\frac{\partial^{2}}{\partial \theta_{r} \partial \theta_{s}} \ell(\boldsymbol{\theta} ; \boldsymbol{X})\right] .
$$

This implies that $n I(\boldsymbol{\theta})$ is the covariance matrix of $\nabla(\ell(\boldsymbol{\theta} ; \boldsymbol{x}))$.

These regularity conditions in the multi-parameter case resemble those in [3] where the parameter is scalar. The following theorem gives the qualitative result for the asymptotic normality of a vector MLE (see [6] for a proof).

Theorem 2.1. Let $\boldsymbol{X}_{\mathbf{1}}, \boldsymbol{X}_{\mathbf{2}}, \ldots, \boldsymbol{X}_{\boldsymbol{n}}$ be i.i.d. random vectors with probability density (mass) functions $f\left(\boldsymbol{x}_{\boldsymbol{i}} \mid \boldsymbol{\theta}\right), 1 \leq i \leq n$, where $\theta \in \Theta \subset \mathbb{R}^{d}$. Also let $\boldsymbol{Z} \sim \mathrm{N}_{d}\left(\mathbf{0}, I_{d \times d}\right)$, where $\mathbf{0}$ is the $d \times 1$ zero vector and $I_{d \times d}$ is the $d \times d$ identity matrix. Then, under (R.C.1)-(R.C.5),

$$
\sqrt{n}\left[I\left(\boldsymbol{\theta}_{\mathbf{0}}\right)\right]^{\frac{1}{2}}\left(\hat{\boldsymbol{\theta}}_{\boldsymbol{n}}(\boldsymbol{X})-\boldsymbol{\theta}_{\mathbf{0}}\right) \underset{n \rightarrow \infty}{\stackrel{\mathrm{d}}{\longrightarrow}} \mathrm{N}_{d}\left(\mathbf{0}, I_{d \times d}\right) .
$$

imsart-bjps ver. 2014/10/16 file: mle_arxiv.tex date: August 10, 2018 
Let us now introduce some notation. We let $C_{b}^{n}\left(\mathbb{R}^{d}\right)$ denote the space of bounded functions on $\mathbb{R}^{d}$ with bounded $k$-th order derivatives for $k \leq n$. We abbreviate $|h|_{1}:=\sup _{i}\left\|\frac{\partial}{\partial x_{i}} h\right\|$ and $|h|_{2}:=\sup _{i, j}\left\|\frac{\partial^{2}}{\partial x_{i} \partial x_{j}} h\right\|$, where $\|\cdot\|=$ $\|\cdot\|_{\infty}$ is the supremum norm. For ease of presentation, we let the subscript $(m)$ denote an index for which $\left|\hat{\theta}_{n}(\boldsymbol{x})_{(m)}-\theta_{0_{(m)}}\right|$ is the largest among the $d$ components:

$(m) \in\{1,2, \ldots, d\}:\left|\hat{\theta}_{n}(\boldsymbol{x})_{(m)}-\theta_{0_{(m)}}\right| \geq\left|\hat{\theta}_{n}(\boldsymbol{x})_{j}-\theta_{0_{j}}\right|, \quad \forall j \in\{1,2, \ldots, d\}$,

and also

$$
Q_{(m)}=Q_{(m)}\left(\boldsymbol{X}, \boldsymbol{\theta}_{\mathbf{0}}\right):=\hat{\theta}_{n}(\boldsymbol{X})_{(m)}-\theta_{0_{(m)}} .
$$

The following theorem gives the main result of this paper.

Theorem 2.2. Let $\boldsymbol{X}_{\mathbf{1}}, \boldsymbol{X}_{\mathbf{2}}, \ldots, \boldsymbol{X}_{\boldsymbol{n}}$ be i.i.d. $\mathbb{R}^{t}$-valued random vectors with probability density (or mass) function $f\left(\boldsymbol{x}_{\boldsymbol{i}} \mid \boldsymbol{\theta}\right)$, for which the parameter space $\Theta$ is an open subset of $\mathbb{R}^{d}$. Let $\boldsymbol{Z} \sim \mathrm{N}_{d}\left(\mathbf{0}, I_{d \times d}\right)$. Assume that the $M L E$ $\hat{\boldsymbol{\theta}}_{\boldsymbol{n}}(\boldsymbol{X})$ exists and is unique and that (R.C.1)-(R.C.5) are satisfied. Let $q: \Theta \rightarrow \mathbb{R}^{d}$ be twice differentiable and $g: \mathbb{R}^{t} \rightarrow \mathbb{R}^{d}$ such that the special structure (1.1) is satisfied. Let

$$
\xi_{i j}=\sum_{l=1}^{d}\left[K^{-\frac{1}{2}}\right]_{k l}\left(g_{l}\left(\boldsymbol{X}_{\boldsymbol{i}}\right)-q_{l}\left(\boldsymbol{\theta}_{\mathbf{0}}\right)\right),
$$

where

$$
K=\left[\nabla q\left(\boldsymbol{\theta}_{\mathbf{0}}\right)\right]\left[I\left(\boldsymbol{\theta}_{\mathbf{0}}\right)\right]^{-1}\left[\nabla q\left(\boldsymbol{\theta}_{\mathbf{0}}\right)\right]^{\top} .
$$

Also, let $Q_{(m)}$ be as in (2.1). Then, for all $h \in C_{b}^{2}\left(\mathbb{R}^{d}\right)$,

$$
\begin{aligned}
& \left|\mathbb{E}\left[h\left(\sqrt{n}\left[I\left(\boldsymbol{\theta}_{\mathbf{0}}\right)\right]^{\frac{1}{2}}\left(\hat{\boldsymbol{\theta}}_{\boldsymbol{n}}(\boldsymbol{X})-\boldsymbol{\theta}_{\mathbf{0}}\right)\right)\right]-\mathbb{E}[h(\boldsymbol{Z})]\right| \\
& \leq \frac{\sqrt{\pi}|h|_{2}}{4 \sqrt{2} n^{3 / 2}} \sum_{i=1}^{n} \sum_{j, k, l=1}^{d}\left\{\mathbb{E}\left|\xi_{i j} \xi_{i k} \xi_{i l}\right|+2\left|\mathbb{E}\left[\xi_{i j} \xi_{i k}\right]\right| \mathbb{E}\left|\xi_{i l}\right|\right\} \\
& \quad+2 \frac{\|h\|_{1}}{\epsilon^{2}}\left[\sum_{j=1}^{d}\left(\hat{\theta}_{n}(\boldsymbol{X})_{j}-\theta_{0_{j}}\right)^{2}\right] \\
& \quad+\frac{|h|_{1}}{\sqrt{n}} \sum_{j=1}^{d}\left\{\mathbb{E}\left[\left|A_{1 j}\left(\boldsymbol{\theta}_{\mathbf{0}}, \boldsymbol{X}\right)\right||| Q_{(m)} \mid<\epsilon\right]+\mathbb{E}\left[\left|A_{2 j}\left(\boldsymbol{\theta}_{\mathbf{0}}, \boldsymbol{X}\right)\right||| Q_{(m)} \mid<\epsilon\right]\right\},
\end{aligned}
$$


where

$$
\begin{aligned}
A_{1 j}\left(\boldsymbol{\theta}_{\mathbf{0}}, \boldsymbol{X}\right)= & n \sum_{k=1}^{d}\left\{\left(\left[I\left(\boldsymbol{\theta}_{\mathbf{0}}\right)\right]^{\frac{1}{2}}\left[\nabla q\left(\boldsymbol{\theta}_{\mathbf{0}}\right)\right]^{-1}-K^{-\frac{1}{2}}\right)_{j k}\left(q_{k}\left(\hat{\boldsymbol{\theta}}_{\boldsymbol{n}}(\boldsymbol{X})\right)-q_{k}\left(\boldsymbol{\theta}_{\mathbf{0}}\right)\right)\right\} \\
A_{2 j}\left(\boldsymbol{\theta}_{\mathbf{0}}, \boldsymbol{X}\right)= & \frac{n}{2} \sum_{k=1}^{d}\left\{\left(\left[I\left(\boldsymbol{\theta}_{\mathbf{0}}\right)\right]^{\frac{1}{2}}\left[\nabla q\left(\boldsymbol{\theta}_{\mathbf{0}}\right)\right]^{-1}\right)_{j k} \sum_{m=1}^{d} \sum_{l=1}^{d}\left(\hat{\theta}_{n}(\boldsymbol{X})_{m}-\theta_{0_{m}}\right)\right. \\
& \left.\times\left(\hat{\theta}_{n}(\boldsymbol{X})_{l}-\theta_{0_{l}}\right) M_{k l m}(\boldsymbol{X})\right\} .
\end{aligned}
$$

Remark 2.3. 1. For fixed $d$, it is clear that the first term is $O\left(n^{-1 / 2}\right)$. For the second term, using [5] we know that the mean squared error (MSE) of the MLE is of order $n^{-1}$. This yields, for fixed $d, \mathbb{E}\left[\sum_{j=1}^{d}\left(\hat{\theta}_{n}(\boldsymbol{X})_{j}-\right.\right.$ $\left.\left.\theta_{0, j}\right)^{2}\right]=O\left(n^{-1}\right)$. To establish that (2.3) is order $n^{-1 / 2}$, it therefore suffices to show that for the third term the quantities $\mathbb{E}\left[\left|A_{1 j}\left(\boldsymbol{\theta}_{\mathbf{0}}, \boldsymbol{X}\right)\right||| Q_{(m)} \mid<\right.$ $\epsilon]$ and $\mathbb{E}\left[\left|A_{2 j}\left(\boldsymbol{\theta}_{\mathbf{0}}, \boldsymbol{X}\right)\right||| Q_{(m)} \mid<\epsilon\right]$ are $O(1)$. For $A_{2 j}\left(\boldsymbol{\theta}_{\mathbf{0}}, \boldsymbol{X}\right)$, we use the Cauchy-Schwarz inequality to get that

$$
\begin{aligned}
& \mathbb{E}\left|\left(\hat{\theta}_{n}(\boldsymbol{X})_{m}-\theta_{0_{m}}\right)\left(\hat{\theta}_{n}(\boldsymbol{X})_{l}-\theta_{0_{l}}\right) M_{j k l}(\boldsymbol{X})\right| \\
& \leq\left[\mathbb{E}\left[\left(\hat{\theta}_{n}(\boldsymbol{X})_{m}-\theta_{0_{m}}\right)^{2}\left(\hat{\theta}_{n}(\boldsymbol{X})_{l}-\theta_{0_{l}}\right)^{2}\right]\right]^{1 / 2} \\
& \quad \times\left[\mathbb{E}\left[\left(M_{k l m}(\boldsymbol{X})\right)^{2}|| Q_{(m)} \mid<\epsilon\right]\right]^{1 / 2} .
\end{aligned}
$$

Since from [5] the MSE is of order $n^{-1}$, we apply (2.4) to the expression of $A_{2 j}\left(\boldsymbol{\theta}_{\mathbf{0}}, \boldsymbol{X}\right)$ to get that $\mathbb{E}\left[\left|A_{2 j}\left(\boldsymbol{\theta}_{\mathbf{0}}, \boldsymbol{X}\right)\right||| Q_{(m)} \mid<\epsilon\right]=O(1)$. Whilst we suspect that $\mathbb{E}\left[\left|A_{1 j}\left(\boldsymbol{\theta}_{\mathbf{0}}, \boldsymbol{X}\right)\right||| Q_{(m)} \mid<\epsilon\right]$ are generally $O(1)$, we have been unable to establish this fact. In the univariate $d=1$ case, this term vanishes (see [2]), and this is also the case in our application in Section 3 (in which case the matrices $I\left(\boldsymbol{\theta}_{\mathbf{0}}\right)$ and $\nabla q\left(\boldsymbol{\theta}_{\mathbf{0}}\right)$ are diagonal).

2. An upper bound on the quantity $\left|\mathbb{E}\left[h\left(\sqrt{n}\left[I\left(\boldsymbol{\theta}_{\mathbf{0}}\right)\right]^{\frac{1}{2}}\left(\hat{\boldsymbol{\theta}}_{\boldsymbol{n}}(\boldsymbol{X})-\boldsymbol{\theta}_{\mathbf{0}}\right)\right)\right]-\mathbb{E}[h(\mathbf{Z})]\right|$ was given in [1]. A brief comparison of that bound and our bound follows. The bound in [1] is more general than our bound. It covers the case of independent but not necessarily identically distributed random vectors. In addition, the bound in [1] is more general in the sense that it is applicable whatever the form of the MLE is; our bound can be used only when (1.1) is satisfied. Furthermore, [1] gives bounds even for cases where the MLE cannot be expressed analytically. On the other hand, our bound is preferable when the MLE has a representation of the form (1.1). Our bound in (2.3) applies to a wider class of test functions $h$; our class is $C_{b}^{2}\left(\mathbb{R}^{d}\right)$, as opposed 
to $C_{b}^{3}\left(\mathbb{R}^{d}\right)$, and has a better dependence on the dimension $d ;$ for fixed $n$, our bound is $O\left(d^{4}\right)$, whereas the bound of [1] is $O\left(d^{6}\right)$. Moreover, in situations where the $M L E$ is already a sum of independent terms our bound is easier to use since if we apply $q(\boldsymbol{x})=\boldsymbol{x}$ to (2.12) yields $R_{2}=0$ in (2.11). This means that our bound (2.3) simplifies to

$$
\begin{aligned}
\mid \mathbb{E} & {\left[h\left(\sqrt{n}\left[I\left(\boldsymbol{\theta}_{\mathbf{0}}\right)\right]^{\frac{1}{2}}\left(\hat{\boldsymbol{\theta}}_{\boldsymbol{n}}(\boldsymbol{X})-\boldsymbol{\theta}_{\mathbf{0}}\right)\right)\right]-\mathbb{E}[h(\boldsymbol{Z})] \mid } \\
& \leq \frac{\sqrt{\pi}|h|_{2}}{4 \sqrt{2} n^{3 / 2}} \sum_{i=1}^{n} \sum_{j, k, l=1}^{d}\left\{\mathbb{E}\left|\xi_{i j} \xi_{i k} \xi_{i l}\right|+2\left|\mathbb{E}\left[\xi_{i j} \xi_{i k}\right]\right| \mathbb{E}\left|\xi_{i l}\right|\right\}
\end{aligned}
$$

Another simplification of the general bound is given in Section 3, where $X_{1}, X_{2}, \ldots, X_{n}$ are taken to be i.i.d. normal random variables with unknown mean and variance, which can also be treated by [1]. The simpler terms in our bound lead to a much improved bound for this example (see Remark 3.2 for more details).

3. If for all $k, m, l \in\{1,2, \ldots, d\},\left|\frac{\partial^{2}}{\partial \theta_{m} \partial \theta_{l}} q_{k}(\boldsymbol{\theta})\right|$ is uniformly bounded in $\Theta$, then we do not need to use $\epsilon$ or conditional expectations in deriving an upper bound (we essentially apply Theorem 2.2 with $\epsilon \rightarrow \infty$ ). This leads to the following simpler bound:

$$
\begin{aligned}
\left|\mathbb{E}\left[h\left(\sqrt{n}\left[I\left(\boldsymbol{\theta}_{\mathbf{0}}\right)\right]^{\frac{1}{2}}\left(\hat{\boldsymbol{\theta}}_{\boldsymbol{n}}(\boldsymbol{X})-\boldsymbol{\theta}_{\mathbf{0}}\right)\right)\right]-\mathbb{E}[h(\boldsymbol{Z})]\right| \\
\leq \frac{\sqrt{\pi}|h|_{2}}{4 \sqrt{2} n^{3 / 2}} \sum_{i=1}^{n} \sum_{j, k, l=1}^{d}\left\{\mathbb{E}\left|\xi_{i j} \xi_{i k} \xi_{i l}\right|+2\left|\mathbb{E}\left[\xi_{i j} \xi_{i k}\right]\right| \mathbb{E}\left|\xi_{i l}\right|\right\} \\
+\frac{|h|_{1}}{\sqrt{n}} \sum_{j=1}^{d}\left\{\mathbb{E}\left|A_{1 j}\left(\boldsymbol{\theta}_{\mathbf{0}}, \boldsymbol{X}\right)\right|+\mathbb{E}\left|A_{2 j}\left(\boldsymbol{\theta}_{\mathbf{0}}, \boldsymbol{X}\right)\right|\right\} .
\end{aligned}
$$

To prove Theorem 2.2, we employ Stein's method and the multivariate delta method. Our strategy consists of benefiting from the special form of $q\left(\hat{\boldsymbol{\theta}}_{\boldsymbol{n}}(\boldsymbol{X})\right)$, which is a sum of random vectors. It is here where multivariate delta method comes into play; instead of comparing $\hat{\boldsymbol{\theta}}_{\boldsymbol{n}}(\boldsymbol{X})$ to $\boldsymbol{Z} \sim$ $\mathrm{N}_{d}\left(\mathbf{0}, I_{d \times d}\right)$, we compare $q\left(\hat{\boldsymbol{\theta}}_{\boldsymbol{n}}(\boldsymbol{X})\right)$ to $\boldsymbol{Z}$ and then find upper bounds on the distributional distance between the distribution of $\hat{\boldsymbol{\theta}}_{\boldsymbol{n}}(\boldsymbol{X})$ and $q\left(\hat{\boldsymbol{\theta}}_{\boldsymbol{n}}(\boldsymbol{X})\right)$. As well as recalling the multivariate delta method, we state and prove two lemmas that will be used in the proof of Theorem 2.2.

Theorem 2.4. (Multivariate delta method) Let the parameter $\boldsymbol{\theta} \in \Theta$, where $\Theta \subset \mathbb{R}^{d}$. Furthermore, let $Y_{n}$ be a sequence of random vectors in $\mathbb{R}^{d}$ 
which, for $\boldsymbol{Z} \sim \mathrm{N}_{d}\left(\mathbf{0}, I_{d \times d}\right)$, satisfies

$$
\sqrt{n}\left(Y_{n}-\boldsymbol{\theta}\right) \underset{n \rightarrow \infty}{\stackrel{\mathrm{d}}{\longrightarrow}} \sum^{\frac{1}{2}} \boldsymbol{Z}
$$

where $\Sigma$ is a symmetric, positive definite covariance matrix. Then, for any function $b: \mathbb{R}^{d} \rightarrow \mathbb{R}^{d}$ of which the Jacobian matrix $\nabla b(\boldsymbol{\theta}) \in \mathbb{R}^{d \times d}$ exists and is invertible, we have that for $\Lambda:=[\nabla b(\boldsymbol{\theta})] \Sigma[\nabla b(\boldsymbol{\theta})]^{\top}$,

$$
\sqrt{n}\left(b\left(Y_{n}\right)-b(\boldsymbol{\theta})\right) \underset{n \rightarrow \infty}{\stackrel{\mathrm{d}}{\longrightarrow}} \Lambda^{\frac{1}{2}} \boldsymbol{Z} .
$$

Lemma 2.5. Let

$$
\boldsymbol{W}=\frac{K^{-\frac{1}{2}}}{\sqrt{n}} \sum_{i=1}^{n}\left[g\left(\boldsymbol{X}_{\boldsymbol{i}}\right)-q\left(\boldsymbol{\theta}_{\mathbf{0}}\right)\right],
$$

where $K$ is as in (2.2). Then $\mathbb{E} \mathbf{W}=\mathbf{0}$ and $\operatorname{Cov}(\mathbf{W})=I_{d \times d}$.

Proof. By the multivariate central limit theorem,

$$
\frac{1}{\sqrt{n}} \sum_{i=1}^{n}\left(g\left(\mathbf{X}_{i}\right)-\mathbb{E} g\left(\mathbf{X}_{1}\right)\right) \underset{n \rightarrow \infty}{\stackrel{\mathrm{d}}{\longrightarrow}} \mathrm{N}_{d}\left(\mathbf{0}, \operatorname{Cov}\left(g\left(\mathbf{X}_{1}\right)\right),\right.
$$

and, by Theorem 2.4,

$$
\sqrt{n}\left(q\left(\hat{\boldsymbol{\theta}}_{\boldsymbol{n}}(\boldsymbol{X})\right)-q\left(\boldsymbol{\theta}_{\mathbf{0}}\right)\right) \underset{n \rightarrow \infty}{\stackrel{\mathrm{d}}{\longrightarrow}} \mathrm{N}_{d}(\mathbf{0}, K) .
$$

Since $q\left(\hat{\boldsymbol{\theta}}_{\boldsymbol{n}}(\boldsymbol{X})\right)=\frac{1}{n} \sum_{i=1}^{n} g\left(\mathbf{X}_{i}\right)$ and $\boldsymbol{X}_{1}, \boldsymbol{X}_{2}, \ldots, \boldsymbol{X}_{n}$ are i.i.d. random vectors, it follows that $\mathbb{E} q\left(\boldsymbol{\theta}_{\mathbf{0}}\right)=\mathbb{E} g\left(\mathbf{X}_{1}\right)$. Furthermore, $K=\operatorname{Cov}\left(g\left(\mathbf{X}_{1}\right)\right)$ because the limiting multivariate normal distributions in (2.7) and (2.8) must have the same mean and covariance. It is now immediate that $\mathbb{E} \mathbf{W}=\mathbf{0}$, and a simple calculation using that $K=\operatorname{Cov}\left(g\left(\mathbf{X}_{1}\right)\right)$ yields that $\operatorname{Cov}(\mathbf{W})=$ $I_{d \times d}$.

Lemma 2.6. Suppose that $X_{1, j}, \ldots, X_{n, j}$ are independent for a fixed $j$, but that the random variables $X_{i, 1}, \ldots, X_{i, d}$ may be dependent for any fixed $i$. For $j=1, \ldots, d$, let $W_{j}=\frac{1}{\sqrt{n}} \sum_{i=1}^{n} X_{i j}$ and denote $\mathbf{W}=\left(W_{1}, \ldots, W_{d}\right)^{\top}$. Suppose that $\mathbb{E}[\mathbf{W}]=\mathbf{0}$ and $\operatorname{Cov}(\mathbf{W})=I_{d \times d}$. Then, for all $h \in C_{b}^{2}\left(\mathbb{R}^{d}\right)$,

$$
\begin{aligned}
|\mathbb{E}[h(\mathbf{W})]-\mathbb{E}[h(\mathbf{Z})]| \leq & \frac{\sqrt{\pi}|h|_{2}}{4 \sqrt{2} n^{3 / 2}} \sum_{i=1}^{n} \sum_{j, k, l=1}^{d}\left\{\mathbb{E}\left|X_{i j} X_{i k} X_{i l}\right|\right. \\
& \left.+2\left|\mathbb{E}\left[X_{i j} X_{i k}\right]\right| \mathbb{E}\left|X_{i l}\right|\right\} .
\end{aligned}
$$


Proof. The following bound follows immediately from Lemma 2.1 of [?]:

$$
\begin{aligned}
|\mathbb{E}[h(\mathbf{W})]-\mathbb{E}[h(\mathbf{Z})]| \leq & \frac{1}{2 n^{3 / 2}}\left\|\frac{\partial^{3} f(\mathbf{x})}{\partial x_{i_{1}} \partial x_{i_{2}} \partial x_{i_{3}}}\right\| \sum_{i=1}^{n} \sum_{j, k, l=1}^{d}\left\{\mathbb{E}\left|X_{i j} X_{i k} X_{i l}\right|\right. \\
& \left.+2\left|\mathbb{E}\left[X_{i j} X_{i k}\right]\right| \mathbb{E}\left|X_{i l}\right|\right\}
\end{aligned}
$$

where $f$ solves the so-called Stein equation

$$
\nabla^{\top} \nabla f(\mathbf{w})-\mathbf{w}^{\top} \nabla f(\mathbf{w})=h(\mathbf{w})-\mathbb{E}[h(\mathbf{Z})] .
$$

From Proposition 2.1 of [?], we have the bound

$$
\left\|\frac{\partial^{3} f(\mathbf{x})}{\partial x_{i_{1}} \partial x_{i_{2}} \partial x_{i_{3}}}\right\| \leq \frac{\sqrt{\pi}}{2 \sqrt{2}}|h|_{2}
$$

which when applied to (2.10) yields (2.9).

Proof of Theorem 2.2. The triangle inequality yields

$$
\left|\mathbb{E}\left[h\left(\sqrt{n}\left[I\left(\boldsymbol{\theta}_{\mathbf{0}}\right)\right]^{\frac{1}{2}}\left(\hat{\boldsymbol{\theta}}_{\boldsymbol{n}}(\boldsymbol{X})-\boldsymbol{\theta}_{\mathbf{0}}\right)\right)\right]-\mathbb{E}[h(\boldsymbol{Z})]\right| \leq R_{1}+R_{2},
$$

where

$$
\begin{aligned}
R_{1} \leq & \left|\mathbb{E}\left[h\left(\sqrt{n} K^{-\frac{1}{2}}\left(q\left(\hat{\boldsymbol{\theta}}_{\boldsymbol{n}}(\boldsymbol{X})\right)-q\left(\boldsymbol{\theta}_{\mathbf{0}}\right)\right)\right)\right]-\mathbb{E}[h(\boldsymbol{Z})]\right|, \\
R_{2} \leq & \mid \mathbb{E}\left[h\left(\sqrt{n}\left[I\left(\boldsymbol{\theta}_{\mathbf{0}}\right)\right]^{\frac{1}{2}}\left(\hat{\boldsymbol{\theta}}_{\boldsymbol{n}}(\boldsymbol{X})-\boldsymbol{\theta}_{\mathbf{0}}\right)\right)\right. \\
& \left.-h\left(\sqrt{n} K^{-\frac{1}{2}}\left(q\left(\hat{\boldsymbol{\theta}}_{\boldsymbol{n}}(\boldsymbol{X})\right)-q\left(\boldsymbol{\theta}_{\mathbf{0}}\right)\right)\right)\right] \mid .
\end{aligned}
$$

The rest of the proof focuses on bounding the terms $R_{1}$ and $R_{2}$.

Step 1: Upper bound for $R_{1}$. For ease of presentation, we denote by

$$
\boldsymbol{W}=\sqrt{n} K^{-\frac{1}{2}}\left(q\left(\hat{\boldsymbol{\theta}}_{\boldsymbol{n}}(\boldsymbol{X})\right)-q\left(\boldsymbol{\theta}_{\mathbf{0}}\right)\right)=\frac{K^{-\frac{1}{2}}}{\sqrt{n}} \sum_{i=1}^{n}\left[g\left(\boldsymbol{X}_{\boldsymbol{i}}\right)-q\left(\boldsymbol{\theta}_{\mathbf{0}}\right)\right],
$$

as in (2.6). Thus, $\boldsymbol{W}=\left(W_{1}, W_{2}, \ldots, W_{d}\right)^{\top}$ with

$$
W_{j}=\frac{1}{\sqrt{n}} \sum_{i=1}^{n} \sum_{l=1}^{d}\left[K^{-\frac{1}{2}}\right]_{j l}\left(g_{l}\left(\boldsymbol{X}_{\boldsymbol{i}}\right)-q_{l}\left(\boldsymbol{\theta}_{\mathbf{0}}\right)\right)=\frac{1}{\sqrt{n}} \sum_{i=1}^{n} \xi_{i j}, \forall j \in\{1, \ldots, d\},
$$

where $\xi_{i j}=\sum_{l=1}^{d}\left[K^{-\frac{1}{2}}\right]_{j l}\left(g_{l}\left(\boldsymbol{X}_{\boldsymbol{i}}\right)-q_{l}\left(\boldsymbol{\theta}_{\mathbf{0}}\right)\right)$ are independent random variables, since the $\boldsymbol{X}_{\boldsymbol{i}}$ are assumed to be independent. Therefore, $W_{j}$ can be 
expressed as a sum of independent terms. This together with Lemma 2.5 ensures that the assumptions of Lemma 2.6 are satisfied. Therefore, (2.9) yields

$$
\begin{aligned}
R_{1} & =|\mathbb{E}[h(\boldsymbol{W})]-\mathbb{E}[h(\boldsymbol{Z})]| \\
& \leq \frac{\sqrt{\pi}|h|_{2}}{4 \sqrt{2} n^{3 / 2}} \sum_{i=1}^{n} \sum_{j, k, l=1}^{d}\left\{\mathbb{E}\left|\xi_{i j} \xi_{i k} \xi_{i l}\right|+2 \mid \mathbb{E}\left[\xi_{i j} \xi_{i k}|\mathbb{E}| \xi_{i l} \mid\right\} .\right.
\end{aligned}
$$

Step 2: Upper bound for $R_{2}$. For ease of presentation, we let

$$
\begin{aligned}
C_{1}:=C_{1}\left(h, q, \boldsymbol{X}, \boldsymbol{\theta}_{\mathbf{0}}\right):= & h\left(\sqrt{n}\left[I\left(\boldsymbol{\theta}_{\mathbf{0}}\right)\right]^{\frac{1}{2}}\left(\hat{\boldsymbol{\theta}}_{\boldsymbol{n}}(\boldsymbol{X})-\boldsymbol{\theta}_{\mathbf{0}}\right)\right) \\
& -h\left(\sqrt{n} K^{-\frac{1}{2}}\left(q\left(\hat{\boldsymbol{\theta}}_{\boldsymbol{n}}(\boldsymbol{X})\right)-q\left(\boldsymbol{\theta}_{\mathbf{0}}\right)\right)\right),
\end{aligned}
$$

and our aim is to find an upper bound for $\left|\mathbb{E}\left[C_{1}\right]\right|$. Let us denote by $[A]_{[j]}$ the $j^{\text {th }}$ row of a matrix $A$. We begin by using a first order Taylor expansion to obtain

$$
\begin{aligned}
h\left(\sqrt{n}\left[I\left(\boldsymbol{\theta}_{\mathbf{0}}\right)\right]^{\frac{1}{2}}\left(\hat{\boldsymbol{\theta}}_{\boldsymbol{n}}(\boldsymbol{x})-\boldsymbol{\theta}_{\mathbf{0}}\right)\right)=h\left(\sqrt{n} K^{-\frac{1}{2}}\left(q\left(\hat{\boldsymbol{\theta}}_{\boldsymbol{n}}(\boldsymbol{X})\right)-q\left(\boldsymbol{\theta}_{\mathbf{0}}\right)\right)\right) \\
+\sum_{j=1}^{d}\left\{\sqrt{n}\left[\left[I\left(\boldsymbol{\theta}_{\mathbf{0}}\right)\right]^{\frac{1}{2}}\right]_{[j]}\left(\hat{\boldsymbol{\theta}}_{\boldsymbol{n}}(\boldsymbol{x})-\boldsymbol{\theta}_{\mathbf{0}}\right)\right. \\
\left.-\sqrt{n}\left[K^{-\frac{1}{2}}\right]_{[j]}\left(q\left(\hat{\boldsymbol{\theta}}_{\boldsymbol{n}}(\boldsymbol{X})\right)-q\left(\boldsymbol{\theta}_{\mathbf{0}}\right)\right)\right\} \frac{\partial}{\partial x_{j}} h(t(\boldsymbol{x})),
\end{aligned}
$$

where $t(\boldsymbol{x})$ is between $\sqrt{n}\left[I\left(\boldsymbol{\theta}_{\mathbf{0}}\right)\right]^{\frac{1}{2}}\left(\hat{\boldsymbol{\theta}}_{\boldsymbol{n}}(\boldsymbol{x})-\boldsymbol{\theta}_{\mathbf{0}}\right)$ and $\sqrt{n} K^{-\frac{1}{2}}\left(q\left(\hat{\boldsymbol{\theta}}_{\boldsymbol{n}}(\boldsymbol{X})\right)-\right.$ $\left.q\left(\boldsymbol{\theta}_{\mathbf{0}}\right)\right)$. Rearranging the terms gives

$$
\begin{aligned}
\left|C_{1}\right| \leq & |h|_{1} \mid \sum_{j=1}^{d} \sqrt{n}\left[\left[I\left(\boldsymbol{\theta}_{\mathbf{0}}\right)\right]^{\frac{1}{2}}\right]_{[j]}\left(\hat{\boldsymbol{\theta}}_{\boldsymbol{n}}(\boldsymbol{x})-\boldsymbol{\theta}_{\mathbf{0}}\right) \\
& -\sqrt{n}\left[K^{-\frac{1}{2}}\right]_{[j]}\left(q\left(\hat{\boldsymbol{\theta}}_{\boldsymbol{n}}(\boldsymbol{X})\right)-q\left(\boldsymbol{\theta}_{\mathbf{0}}\right)\right) \mid .
\end{aligned}
$$

For $\boldsymbol{\theta}_{\mathbf{0}}^{*}$ between $\hat{\boldsymbol{\theta}}_{\boldsymbol{n}}(\boldsymbol{x})$ and $\boldsymbol{\theta}_{\mathbf{0}}$, a second order Taylor expansion of $q_{j}\left(\hat{\boldsymbol{\theta}}_{\boldsymbol{n}}(\boldsymbol{x})\right)$ about $\boldsymbol{\theta}_{\mathbf{0}}$ gives that, for all $j \in\{1,2, \ldots, d\}$,

$$
\begin{aligned}
q_{j}\left(\hat{\boldsymbol{\theta}}_{\boldsymbol{n}}(\boldsymbol{x})\right)= & q_{j}\left(\boldsymbol{\theta}_{\mathbf{0}}\right)+\sum_{k=1}^{d}\left(\hat{\theta}_{n}(\boldsymbol{x})_{k}-\theta_{0_{k}}\right) \frac{\partial}{\partial \theta_{k}} q_{j}\left(\boldsymbol{\theta}_{\mathbf{0}}\right) \\
& +\frac{1}{2} \sum_{k=1}^{d} \sum_{l=1}^{d}\left(\hat{\theta}_{n}(\boldsymbol{x})_{k}-\theta_{0_{k}}\right)\left(\hat{\theta}_{n}(\boldsymbol{x})_{l}-\theta_{0_{l}}\right) \frac{\partial^{2}}{\partial \theta_{k} \partial \theta_{l}} q_{j}\left(\boldsymbol{\theta}_{\mathbf{0}}^{*}\right)
\end{aligned}
$$


and after a small rearrangement of the terms we get that

$$
\begin{aligned}
{\left[\nabla q\left(\boldsymbol{\theta}_{\mathbf{0}}\right)\right]_{[j]}\left(\hat{\boldsymbol{\theta}}_{\boldsymbol{n}}(\boldsymbol{x})\right.} & \left.-\boldsymbol{\theta}_{\mathbf{0}}\right)=q_{j}\left(\hat{\boldsymbol{\theta}}_{\boldsymbol{n}}(\boldsymbol{x})\right)-q_{j}\left(\boldsymbol{\theta}_{\mathbf{0}}\right) \\
& -\frac{1}{2} \sum_{k=1}^{d} \sum_{l=1}^{d}\left(\hat{\theta}_{n}(\boldsymbol{x})_{k}-\theta_{0_{k}}\right)\left(\hat{\theta}_{n}(\boldsymbol{x})_{l}-\theta_{0_{l}}\right) \frac{\partial^{2}}{\partial \theta_{k} \partial \theta_{l}} q_{j}\left(\boldsymbol{\theta}_{\mathbf{0}}^{*}\right) .
\end{aligned}
$$

Since this holds for all $j \in\{1,2, \ldots, d\}$, we have that

$$
\begin{aligned}
\nabla q\left(\boldsymbol{\theta}_{\mathbf{0}}\right)\left(\hat{\boldsymbol{\theta}}_{\boldsymbol{n}}(\boldsymbol{x})\right. & \left.-\boldsymbol{\theta}_{\mathbf{0}}\right)=q\left(\hat{\boldsymbol{\theta}}_{\boldsymbol{n}}(\boldsymbol{X})\right)-q\left(\boldsymbol{\theta}_{\mathbf{0}}\right) \\
& -\frac{1}{2} \sum_{k=1}^{d} \sum_{l=1}^{d}\left(\hat{\theta}_{n}(\boldsymbol{x})_{k}-\theta_{0_{k}}\right)\left(\hat{\theta}_{n}(\boldsymbol{x})_{l}-\theta_{0_{l}}\right) \frac{\partial^{2}}{\partial \theta_{k} \partial \theta_{l}} q\left(\boldsymbol{\theta}_{\mathbf{0}}^{*}\right),
\end{aligned}
$$

which then leads to

$$
\begin{aligned}
& \sqrt{n}\left[I\left(\boldsymbol{\theta}_{\mathbf{0}}\right)\right]^{\frac{1}{2}}\left(\hat{\boldsymbol{\theta}}_{\boldsymbol{n}}(\boldsymbol{X})-\boldsymbol{\theta}_{\mathbf{0}}\right)=\sqrt{n}\left[I\left(\boldsymbol{\theta}_{\mathbf{0}}\right)\right]^{\frac{1}{2}}\left[\nabla q\left(\boldsymbol{\theta}_{\mathbf{0}}\right)\right]^{-1}\left(q\left(\hat{\boldsymbol{\theta}}_{\boldsymbol{n}}(\boldsymbol{X})\right)-q\left(\boldsymbol{\theta}_{\mathbf{0}}\right)\right) \\
& -\frac{\sqrt{n}}{2}\left[I\left(\boldsymbol{\theta}_{\mathbf{0}}\right)\right]^{\frac{1}{2}}\left[\nabla q\left(\boldsymbol{\theta}_{\mathbf{0}}\right)\right]^{-1} \sum_{k=1}^{d} \sum_{l=1}^{d}\left(\hat{\theta}_{n}(\boldsymbol{x})_{k}-\theta_{0_{k}}\right)\left(\hat{\theta}_{n}(\boldsymbol{x})_{l}-\theta_{0_{l}}\right) \frac{\partial^{2}}{\partial \theta_{k} \partial \theta_{l}} q\left(\boldsymbol{\theta}_{\mathbf{0}}^{*}\right) .
\end{aligned}
$$

Subtracting the quantity $\sqrt{n} K^{-\frac{1}{2}}\left(q\left(\hat{\boldsymbol{\theta}}_{\boldsymbol{n}}(\boldsymbol{X})\right)-q\left(\boldsymbol{\theta}_{\mathbf{0}}\right)\right)$ from both sides of (2.14) now gives

$$
\begin{aligned}
& \sqrt{n}\left[I\left(\boldsymbol{\theta}_{\mathbf{0}}\right)\right]^{\frac{1}{2}}\left(\hat{\boldsymbol{\theta}}_{\boldsymbol{n}}(\boldsymbol{X})-\boldsymbol{\theta}_{\mathbf{0}}\right)-\sqrt{n} K^{-\frac{1}{2}}\left(q\left(\hat{\boldsymbol{\theta}}_{\boldsymbol{n}}(\boldsymbol{X})\right)-q\left(\boldsymbol{\theta}_{\mathbf{0}}\right)\right) \\
& =\sqrt{n}\left(\left[I\left(\boldsymbol{\theta}_{\mathbf{0}}\right)\right]^{\frac{1}{2}}\left[\nabla q\left(\boldsymbol{\theta}_{\mathbf{0}}\right)\right]^{-1}-K^{-\frac{1}{2}}\right)\left(q\left(\hat{\boldsymbol{\theta}}_{\boldsymbol{n}}(\boldsymbol{X})\right)-q\left(\boldsymbol{\theta}_{\mathbf{0}}\right)\right) \\
& -\frac{\sqrt{n}}{2}\left[I\left(\boldsymbol{\theta}_{\mathbf{0}}\right)\right]^{\frac{1}{2}}\left[\nabla q\left(\boldsymbol{\theta}_{\mathbf{0}}\right)\right]^{-1} \sum_{k=1}^{d} \sum_{l=1}^{d}\left(\hat{\theta}_{n}(\boldsymbol{x})_{k}-\theta_{0_{k}}\right)\left(\hat{\theta}_{n}(\boldsymbol{x})_{l}-\theta_{0_{l}}\right) \frac{\partial^{2}}{\partial \theta_{k} \partial \theta_{l}} q\left(\boldsymbol{\theta}_{\mathbf{0}}^{*}\right) . \\
& \quad \text { imsart-bjps ver. 2014/10/16 file: mle_arxiv.tex date: August 10, } 2018
\end{aligned}
$$


Componentwise we have that

$$
\begin{aligned}
& \sqrt{n}\left[\left[I\left(\boldsymbol{\theta}_{\mathbf{0}}\right)\right]^{\frac{1}{2}}\right]_{[j]}\left(\hat{\boldsymbol{\theta}}_{\boldsymbol{n}}(\boldsymbol{X})-\boldsymbol{\theta}_{\mathbf{0}}\right)-\sqrt{n}\left[K^{-\frac{1}{2}}\right]_{[j]}\left(q\left(\hat{\boldsymbol{\theta}}_{\boldsymbol{n}}(\boldsymbol{X})\right)-q\left(\boldsymbol{\theta}_{\mathbf{0}}\right)\right) \\
= & \sqrt{n}\left(\left[\left[I\left(\boldsymbol{\theta}_{\mathbf{0}}\right)\right]^{\frac{1}{2}}\left[\nabla q\left(\boldsymbol{\theta}_{\mathbf{0}}\right)\right]^{-1}\right]_{[j]}-\left[K^{-\frac{1}{2}}\right]_{[j]}\right)\left(q\left(\hat{\boldsymbol{\theta}}_{\boldsymbol{n}}(\boldsymbol{X})\right)-q\left(\boldsymbol{\theta}_{\mathbf{0}}\right)\right) \\
& -\frac{\sqrt{n}}{2}\left[\left[I\left(\boldsymbol{\theta}_{\mathbf{0}}\right)\right]^{\frac{1}{2}}\left[\nabla q\left(\boldsymbol{\theta}_{\mathbf{0}}\right)\right]^{-1}\right]_{[j]} \\
& \times \sum_{k=1}^{d} \sum_{l=1}^{d}\left(\hat{\theta}_{n}(\boldsymbol{x})_{k}-\theta_{0_{k}}\right)\left(\hat{\theta}_{n}(\boldsymbol{x})_{l}-\theta_{0_{l}}\right) \frac{\partial^{2}}{\partial \theta_{k} \partial \theta_{l}} q\left(\boldsymbol{\theta}_{\mathbf{0}}^{*}\right) \\
= & \sqrt{n} \sum_{k=1}^{d}\left\{\left(\left[I\left(\boldsymbol{\theta}_{\mathbf{0}}\right)\right]^{\frac{1}{2}}\left[\nabla q\left(\boldsymbol{\theta}_{\mathbf{0}}\right)\right]^{-1}-K^{-\frac{1}{2}}\right)_{j k}\left(q_{k}\left(\hat{\boldsymbol{\theta}}_{\boldsymbol{n}}(\boldsymbol{X})\right)-q_{k}\left(\boldsymbol{\theta}_{\mathbf{0}}\right)\right)\right\} \\
& -\frac{\sqrt{n}}{2} \sum_{k=1}^{d}\left\{\left(\left[I\left(\boldsymbol{\theta}_{\mathbf{0}}\right)\right]^{\frac{1}{2}}\left[\nabla q\left(\boldsymbol{\theta}_{\mathbf{0}}\right)\right]^{-1}\right)_{j k}\right. \\
& \left.\times \sum_{m=1}^{d} \sum_{l=1}^{d}\left(\hat{\theta}_{n}(\boldsymbol{x})_{m}-\theta_{0_{m}}\right)\left(\hat{\theta}_{n}(\boldsymbol{x})_{l}-\theta_{0_{l}}\right) \frac{\partial^{2}}{\partial \theta_{m} \partial \theta_{l}} q_{k}\left(\boldsymbol{\theta}_{\mathbf{0}}^{*}\right)\right\} .
\end{aligned}
$$

We need to take into account that $\left|\frac{\partial^{2}}{\partial \theta_{k} \partial \theta_{l}} q_{j}(\boldsymbol{\theta})\right|$ is not uniformly bounded in $\boldsymbol{\theta}$. However, due to (R.C.3), it is assumed that for any $\boldsymbol{\theta}_{\mathbf{0}} \in \Theta$ there exists $0<\epsilon=\epsilon\left(\boldsymbol{\theta}_{\mathbf{0}}\right)$ and constants $M_{j k l}(\boldsymbol{X}), \forall j, k, l \in\{1,2, \ldots, d\}$, such that $\left|\frac{\partial^{2}}{\partial \theta_{k} \partial \theta_{l}} q_{j}(\boldsymbol{\theta})\right| \leq M_{j k l}(\boldsymbol{X})$ for all $\boldsymbol{\theta} \in \Theta$ such that $\left|\theta_{j}-\theta_{0_{j}}\right|<\epsilon, \forall j \in$ $\{1,2, \ldots, d\}$. Therefore, with $\epsilon>0$ and $Q_{(m)}$ as in (2.1), the law of total expectation yields

$$
\begin{aligned}
R_{2}=\left|\mathbb{E}\left[C_{1}\right]\right| \leq \mathbb{E}\left|C_{1}\right|= & \mathbb{E}\left[\left|C_{1}\right||| Q_{(m)} \mid \geq \epsilon\right] \mathbb{P}\left(\left|Q_{(m)}\right| \geq \epsilon\right) \\
& +\mathbb{E}\left[\left|C_{1}\right||| Q_{(m)} \mid<\epsilon\right] \mathbb{P}\left(\left|Q_{(m)}\right|<\epsilon\right) .
\end{aligned}
$$

The Markov inequality applied to $\mathbb{P}\left(\left|Q_{(m)}\right| \geq \epsilon\right)$ yields

$$
\begin{aligned}
\mathbb{P}\left(\left|Q_{(m)}\right| \geq \epsilon\right) & =\mathbb{P}\left(\left|\hat{\theta}_{n}(\boldsymbol{X})_{(m)}-\theta_{0_{(m)}}\right| \geq \epsilon\right) \\
& \leq \mathbb{P}\left(\sum_{j=1}^{d}\left(\hat{\theta}_{n}(\boldsymbol{X})_{j}-\theta_{0_{j}}\right)^{2} \geq \epsilon^{2}\right) \\
& \leq \frac{1}{\epsilon^{2}} \mathbb{E}\left[\sum_{j=1}^{d}\left(\hat{\theta}_{n}(\boldsymbol{X})_{j}-\theta_{0_{j}}\right)^{2}\right] .
\end{aligned}
$$


In addition, $\mathbb{P}\left(\left|Q_{(m)}\right|<\epsilon\right) \leq 1$, which is a reasonable bound because the consistency of the MLE, a result of (R.C.1)-(R.C.5), ensures that $\mid \hat{\theta}_{n}(\boldsymbol{x})_{(m)}-$ $\theta_{0_{(m)}} \mid$ should be small and therefore $\mathbb{P}\left(\left|Q_{(m)}\right|<\epsilon\right)=\mathbb{P}\left(\left|\hat{\theta}_{n}(\boldsymbol{x})_{(m)}-\theta_{0_{(m)}}\right|<\right.$ $\epsilon) \approx 1$. This result along with (2.17) applied to (2.16) gives

$$
R_{2} \leq 2 \frac{\|h\|}{\epsilon^{2}} \mathbb{E}\left[\sum_{j=1}^{d}\left(\hat{\theta}_{n}(\boldsymbol{X})_{j}-\theta_{0_{j}}\right)^{2}\right]+\mathbb{E}\left[\left|C_{1}\right||| Q_{(m)} \mid<\epsilon\right] .
$$

For an upper bound on $\mathbb{E}\left[\left|C_{1}\right||| Q_{(m)} \mid<\epsilon\right]$, using (2.13) and (2.15) yields

$$
\begin{aligned}
R_{2} \leq & 2 \frac{\|h\|}{\epsilon^{2}} \mathbb{E}\left[\sum_{j=1}^{d}\left(\hat{\theta}_{n}(\boldsymbol{X})_{j}-\theta_{0_{j}}\right)^{2}\right]+\frac{|h|_{1}}{\sqrt{n}} \sum_{j=1}^{d}\left\{\mathbb{E}\left[\left|A_{1 j}\left(\boldsymbol{\theta}_{\mathbf{0}}, \boldsymbol{X}\right)\right||| Q_{(m)} \mid<\epsilon\right]\right. \\
& \left.+\mathbb{E}\left[\left|A_{2 j}\left(\boldsymbol{\theta}_{\mathbf{0}}, \boldsymbol{X}\right)\right||| Q_{(m)} \mid<\epsilon\right]\right\},
\end{aligned}
$$

with $A_{1 j}\left(\boldsymbol{\theta}_{\mathbf{0}}, \boldsymbol{X}\right)$ and $A_{2 j}\left(\boldsymbol{\theta}_{\mathbf{0}}, \boldsymbol{X}\right)$ given as in the statement of the theorem. Summing our bounds for $R_{1}$ and $R_{2}$ gives the assertion of the theorem.

\section{Normally distributed random variables}

In this section, we illustrate the application of the general bound of Theorem 2.2 by considering the important case that $X_{1}, X_{2}, \ldots, X_{n}$ are i.i.d. $\mathrm{N}\left(\mu, \sigma^{2}\right)$ random variables with $\boldsymbol{\theta}_{\mathbf{0}}=\left(\mu, \sigma^{2}\right)$. Here the density function is

$$
f(x \mid \boldsymbol{\theta})=\frac{1}{\sqrt{2 \pi \sigma^{2}}} \exp \left\{-\frac{1}{2 \sigma^{2}}(x-\mu)^{2}\right\}, \quad x \in \mathbb{R} .
$$

It is well-known that in this case the MLE exists, is unique and equal to $\hat{\boldsymbol{\theta}}_{\boldsymbol{n}}(\boldsymbol{X})=\left(\bar{X}, \frac{1}{n} \sum_{i=1}^{n}\left(X_{i}-\bar{X}\right)^{2}\right)^{\top} ;$ see for example [6, p.118]. As we shall see in the proof of the following corollary, the MLE has a representation of the form (1.1). Thus, Theorem 2.2 can be applied to derive the following bound. Empirical results are given after the proof.

Corollary 3.1. Let $X_{1}, X_{2}, \ldots, X_{n}$ be i.i.d. $\mathrm{N}\left(\mu, \sigma^{2}\right)$ random variables and let $\boldsymbol{Z} \sim \mathrm{N}_{2}\left(\mathbf{0}, I_{2 \times 2}\right)$. Then, for all $h \in C_{b}^{2}\left(\mathbb{R}^{2}\right)$,

$$
\left|\mathbb{E}\left[h\left(\sqrt{n}\left[I\left(\boldsymbol{\theta}_{\mathbf{0}}\right)\right]^{\frac{1}{2}}\left(\hat{\boldsymbol{\theta}}_{\boldsymbol{n}}(\boldsymbol{X})-\boldsymbol{\theta}_{\mathbf{0}}\right)\right)\right]-\mathbb{E}[h(\boldsymbol{Z})]\right| \leq \frac{7|h|_{2}}{\sqrt{n}}+\frac{|h|_{1}}{\sqrt{2 n}} .
$$

Remark 3.2. An upper bound for the distributional distance between the $M L E$ of the normal distribution, treated under canonical parametrisation, 
with unknown mean and variance has also been obtained in [1]. That bound is also of order $O\left(n^{-1 / 2}\right)$, but our numerical constants are two orders of magnitude smaller. Furthermore, our test functions $h$ are less restrictive, coming from the class $C_{b}^{2}\left(\mathbb{R}^{2}\right)$ rather than the class $C_{b}^{3}\left(\mathbb{R}^{2}\right)$. Finally, the bound in (3.2) does not depend on the parameter $\boldsymbol{\theta}_{\mathbf{0}}=\left(\mu, \sigma^{2}\right)$, while the bound given in [1] depends on the natural parameters and blows up when the variance tends to zero or infinity.

Proof. From the general approach in the previous section, we want to have $q: \Theta \rightarrow \mathbb{R}^{2}$, such that

$$
q\left(\hat{\boldsymbol{\theta}}_{\boldsymbol{n}}(\boldsymbol{x})\right)=\frac{1}{n} \sum_{i=1}^{n}\left(\begin{array}{l}
g_{1}\left(x_{i}\right) \\
g_{2}\left(x_{i}\right)
\end{array}\right)
$$

We take

$$
q(\boldsymbol{\theta})=q\left(\theta_{1}, \theta_{2}\right)=\left(\theta_{1}, \theta_{2}+\left(\theta_{1}-\mu\right)^{2}\right)^{\top},
$$

which then yields

$$
\begin{aligned}
q\left(\hat{\boldsymbol{\theta}}_{n}(\boldsymbol{X})\right) & =q\left(\bar{X}, \frac{1}{n} \sum_{i=1}^{n}\left(X_{i}-\bar{X}\right)^{2}\right)^{\top}=\left(\bar{X}, \frac{1}{n} \sum_{i=1}^{n}\left(X_{i}-\bar{X}\right)^{2}+(\bar{X}-\mu)^{2}\right)^{\top} \\
& =\left(\frac{1}{n} \sum_{i=1}^{n} X_{i}, \frac{1}{n} \sum_{i=1}^{n}\left(X_{i}-\mu\right)^{2}\right)^{\top}
\end{aligned}
$$

and therefore

$$
g_{1}\left(x_{i}\right)=x_{i}, \quad g_{2}\left(x_{i}\right)=\left(x_{i}-\mu\right)^{2},
$$

and thus the MLE has a representation of the form (1.1). is

We now note that the Jacobian matrix of $q(\boldsymbol{\theta})$ evaluated at $\boldsymbol{\theta}_{\mathbf{0}}=\left(\mu, \sigma^{2}\right)$

$$
\nabla q\left(\boldsymbol{\theta}_{\mathbf{0}}\right)=\left(\begin{array}{ll}
1 & 0 \\
0 & 1
\end{array}\right)
$$

Furthermore, it is easily deduced from (3.3) that $\forall k, m, l \in\{1,2\}$, we have that $\left|\frac{\partial^{2}}{\partial \theta_{m} \partial \theta_{l}} q_{k}(\boldsymbol{\theta})\right| \leq 2$. Hence, using Remark 2.3 we conclude that the general bound is as in (2.5) and no positive constant $\epsilon$ is necessary. Now, for $l\left(\boldsymbol{\theta}_{\mathbf{0}} ; \boldsymbol{X}\right)$ denoting the log-likelihood function, we obtain from (3.1) that the expected Fisher information matrix is

$$
I\left(\boldsymbol{\theta}_{\mathbf{0}}\right)=-\frac{1}{n} \mathbb{E}\left[\nabla^{\top} \nabla\left(l\left(\boldsymbol{\theta}_{\mathbf{0}} ; \boldsymbol{X}\right)\right)\right]=\left(\begin{array}{cc}
\frac{1}{\sigma^{2}} & 0 \\
0 & \frac{1}{2 \sigma^{4}}
\end{array}\right) .
$$


The results in (3.6) and (3.7) yield

$$
K=\left[\nabla q\left(\boldsymbol{\theta}_{\mathbf{0}}\right)\right]\left[I\left(\boldsymbol{\theta}_{\mathbf{0}}\right)\right]^{-1}\left[\nabla q\left(\boldsymbol{\theta}_{\mathbf{0}}\right)\right]^{\top}=\left[I\left(\boldsymbol{\theta}_{\mathbf{0}}\right)\right]^{-1}=\left(\begin{array}{cc}
\sigma^{2} & 0 \\
0 & 2 \sigma^{4}
\end{array}\right) .
$$

Using (2.6), (3.4), (3.5) and (3.8), we get that

$$
\begin{aligned}
\boldsymbol{W} & =\frac{K^{-\frac{1}{2}}}{\sqrt{n}} \sum_{i=1}^{n}\left[g\left(X_{i}\right)-q\left(\boldsymbol{\theta}_{\mathbf{0}}\right)\right]=\frac{1}{\sqrt{n}}\left(\begin{array}{cc}
\frac{1}{\sigma} & 0 \\
0 & \frac{1}{\sqrt{2} \sigma^{2}}
\end{array}\right) \sum_{i=1}^{n}\left(\begin{array}{c}
X_{i}-\mu \\
\left(X_{i}-\mu\right)^{2}-\sigma^{2}
\end{array}\right) \\
& =\left(\begin{array}{c}
\sum_{i=1}^{n} \frac{X_{i}-\mu}{\sigma \sqrt{n}} \\
\sum_{i=1}^{n} \frac{\left(X_{i}-\mu\right)^{2}-\sigma^{2}}{\sqrt{2 n} \sigma^{2}}
\end{array}\right),
\end{aligned}
$$

and therefore, for $i \in\{1, \ldots, n\}$,

$$
\xi_{i 1}=\frac{X_{i}-\mu}{\sigma}, \quad \xi_{i 2}=\frac{\left(X_{i}-\mu\right)^{2}-\sigma^{2}}{\sqrt{2} \sigma^{2}} .
$$

For the first term of the general upper bound in (2.5), we are required to bound certain expectations involving $\xi_{i j}$. We first note that $\frac{X_{i}-\mu}{\sigma} \stackrel{\mathrm{d}}{=} Z \sim$ $\mathrm{N}(0,1)$. Using the standard formulas for the moments and absolute moments of the standard normal distribution (see [10]), we have, for all $i=1, \ldots, n$,

$$
\mathbb{E}\left|\xi_{i 1}\right|=\mathbb{E}|Z|=\sqrt{\frac{2}{\pi}}, \quad \mathbb{E} \xi_{i 1}^{2}=\mathbb{E} Z^{2}=1, \quad \mathbb{E}\left|\xi_{i 1}\right|^{3}=\mathbb{E}|Z|^{3}=2 \sqrt{\frac{2}{\pi}}
$$

and, by Hölder's inequality,

$$
\begin{aligned}
\mathbb{E}\left|\xi_{i 2}\right| & =\frac{1}{\sqrt{2}} \mathbb{E}\left|Z^{2}-1\right| \leq \frac{1}{\sqrt{2}}\left(\mathbb{E}\left(Z^{2}-1\right)^{2}\right)^{1 / 2}=1 \\
\mathbb{E} \xi_{i 2}^{2} & =\frac{1}{2} \mathbb{E}\left(Z^{2}-1\right)^{2}=1 \\
\mathbb{E}\left|\xi_{i 2}\right|^{3} & =\frac{1}{2^{3 / 2}} \mathbb{E}\left|Z^{2}-1\right|^{3} \leq \frac{1}{2^{3 / 2}}\left(\mathbb{E}\left(Z^{2}-1\right)^{4}\right)^{3 / 4} \\
& =\frac{1}{2^{3 / 2}}\left(\mathbb{E} Z^{8}-4 \mathbb{E} Z^{6}+6 \mathbb{E} Z^{4}-4 \mathbb{E} Z^{2}+1\right)^{3 / 4} \\
& =\frac{1}{2^{3 / 2}}(105-60+18-4+1)^{3 / 4}=15^{3 / 4}
\end{aligned}
$$

Therefore,

$$
\begin{aligned}
\sum_{i=1}^{n} \sum_{j, k, l}^{2} \mathbb{E}\left|\xi_{i j} \xi_{i k} \xi_{i l}\right| & =\sum_{i=1}^{n}\left(\mathbb{E}\left|\xi_{i 1}\right|^{3}+3 \mathbb{E} \xi_{i 1}^{2} \mathbb{E}\left|\xi_{i 2}\right|+3 \mathbb{E}\left|\xi_{i 1}\right| \mathbb{E} \xi_{i 2}^{2}+\mathbb{E}\left|\xi_{i 2}\right|^{3}\right) \\
& \leq \sum_{i=1}^{n}\left(2 \sqrt{\frac{2}{\pi}}+3+3 \sqrt{\frac{2}{\pi}}+15^{3 / 4}\right)<14.612 n
\end{aligned}
$$


and

$$
\sum_{i=1}^{n} \sum_{j, k, l=1}^{2}\left|\mathbb{E} \xi_{i j} \xi_{i k}\right| \mathbb{E}\left|\xi_{i l}\right|=\sum_{i=1}^{n} \sum_{j, l=1}^{2} \mathbb{E} \xi_{i j}^{2} \mathbb{E}\left|\xi_{i l}\right|=2 n\left(1+\sqrt{\frac{2}{\pi}}\right) .
$$

Applying the results of (3.9) and (3.10) to the first term of the general bound in $(2.5)$ yields

$$
\begin{aligned}
& \frac{\sqrt{\pi}|h|_{2}}{4 \sqrt{2} n^{3 / 2}} \sum_{i=1}^{n} \sum_{j, k, l=1}^{2}\left\{\mathbb{E}\left|\xi_{i j} \xi_{i k} \xi_{i l}\right|+2\left|\mathbb{E} \xi_{i j} \xi_{i k}\right| \mathbb{E}\left|\xi_{i l}\right|\right\} \\
& \quad \leq \frac{\sqrt{\pi}|h|_{2}}{4 \sqrt{2} n^{3 / 2}}\left(4 n\left(1+\sqrt{\frac{2}{\pi}}\right)+14.612 n\right)=\frac{6.833|h|_{2}}{\sqrt{n}} \leq \frac{7|h|_{2}}{\sqrt{n}} .
\end{aligned}
$$

For the second term, we need to calculate $A_{1 j}\left(\boldsymbol{\theta}_{\mathbf{0}}, \boldsymbol{X}\right)$ and $A_{2 j}\left(\boldsymbol{\theta}_{\mathbf{0}}, \boldsymbol{X}\right)$ as in Theorem 2.2. The results of (3.6) and (3.8) yield to

$$
\left[I\left(\boldsymbol{\theta}_{\mathbf{0}}\right)\right]^{\frac{1}{2}}\left[\nabla\left(q\left(\boldsymbol{\theta}_{\mathbf{0}}\right)\right)\right]^{-1}-K^{-\frac{1}{2}}=\mathbf{0}_{2 \times 2},
$$

where $\mathbf{0}_{2 \times 2}$ denotes the 2 by 2 zero matrix. Therefore $A_{1 j}\left(\boldsymbol{\theta}_{\mathbf{0}}, \boldsymbol{X}\right)=0$ for $j=1,2$. In order to calculate $A_{2 j}\left(\boldsymbol{\theta}_{\mathbf{0}}, \boldsymbol{X}\right)$, we note that

$$
\begin{aligned}
& \frac{\partial^{2}}{\partial \theta_{m} \partial \theta_{l}} q_{1}(\boldsymbol{\theta})=0, \quad \forall m, l \in\{1,2\} \\
& \frac{\partial^{2}}{\partial \theta_{1} \partial \theta_{2}} q_{2}(\boldsymbol{\theta})=\frac{\partial^{2}}{\partial \theta_{2} \partial \theta_{1}} q_{2}(\boldsymbol{\theta})=\frac{\partial^{2}}{\partial \theta_{2}^{2}} q_{2}(\boldsymbol{\theta})=0, \\
& \frac{\partial^{2}}{\partial \theta_{1}^{2}} q_{2}(\boldsymbol{\theta})=2 .
\end{aligned}
$$

Using this result as well as (3.6) and (3.7), we get that

$$
\begin{aligned}
& A_{21}\left(\boldsymbol{\theta}_{\mathbf{0}}, \boldsymbol{X}\right)=0, \\
& A_{22}\left(\boldsymbol{\theta}_{\mathbf{0}}, \boldsymbol{X}\right)=\frac{n}{2 \sqrt{2} \sigma^{2}} 2(\bar{X}-\mu)^{2}=\frac{n}{\sqrt{2} \sigma^{2}}(\bar{X}-\mu)^{2} .
\end{aligned}
$$

Therefore the last term of the general upper bound in (2.5) becomes

$$
\begin{aligned}
& \frac{|h|_{1}}{\sqrt{n}} \sum_{j=1}^{2}\left\{\mathbb{E}\left|A_{1 j}\left(\boldsymbol{\theta}_{\mathbf{0}}, \boldsymbol{X}\right)\right|+\mathbb{E}\left|A_{2 j}\left(\boldsymbol{\theta}_{\mathbf{0}}, \boldsymbol{X}\right)\right|\right\} \\
& \quad=\frac{|h|_{1}}{\sqrt{n}} \mathbb{E}\left|A_{22}\left(\boldsymbol{\theta}_{\mathbf{0}}, \boldsymbol{X}\right)\right|=\frac{\sqrt{n}}{\sqrt{2} \sigma^{2}}|h|_{1} \mathbb{E}\left[(\bar{X}-\mu)^{2}\right]=\frac{|h|_{1}}{\sqrt{2 n}} .
\end{aligned}
$$

Finally, the bounds in (3.11) and (3.12) are applied to (2.5) to obtain the assertion of the corollary. 
Here, we carry out a large-scale simulation study to investigate the accuracy of the bound in (3.2). For $n=10^{j}, j=3,4,5,6$, we start by generating $10^{4}$ trials of $n$ random independent observations, $X$, following the $\mathrm{N}\left(\mu, \sigma^{2}\right)$ distribution. We take $\mu=1, \sigma^{2}=1$ for our simulations. Then $\sqrt{n}\left[I\left(\boldsymbol{\theta}_{\mathbf{0}}\right)\right]^{\frac{1}{2}}\left(\hat{\boldsymbol{\theta}}_{\boldsymbol{n}}(\boldsymbol{X})-\boldsymbol{\theta}_{\mathbf{0}}\right)$ is evaluated in each trial, which in turn gives a vector of $10^{4}$ values. The function $h(x, y)=\left(x^{2}+y^{2}+1\right)^{-1}$, which belongs in $C_{b}^{2}\left(\mathbb{R}^{2}\right)$, is then applied to these values in order to get the sample mean, which we denote by $\hat{\mathbb{E}}\left[h\left(\sqrt{n}\left[I\left(\boldsymbol{\theta}_{\mathbf{0}}\right)\right]^{\frac{1}{2}}\left(\hat{\boldsymbol{\theta}}_{\boldsymbol{n}}(\boldsymbol{X})-\boldsymbol{\theta}_{\mathbf{0}}\right)\right)\right]$. For the function $h$, it is straightforward to see that

$$
|h|_{1}=\frac{3 \sqrt{3}}{8}, \quad|h|_{2}=2 .
$$

We use these values to calculate the bound in (3.2). We define

$$
Q_{h}\left(\boldsymbol{\theta}_{\mathbf{0}}\right):=\left|\hat{\mathbb{E}}\left[h\left(\sqrt{n}\left[I\left(\boldsymbol{\theta}_{\mathbf{0}}\right)\right]^{\frac{1}{2}}\left(\hat{\boldsymbol{\theta}}_{\boldsymbol{n}}(\boldsymbol{X})-\boldsymbol{\theta}_{\mathbf{0}}\right)\right)\right]-\tilde{\mathbb{E}}[h(\boldsymbol{Z})]\right|,
$$

where $\tilde{\mathbb{E}}[h(\boldsymbol{Z})]=0.461$ is the approximation of $\mathbb{E}[h(\boldsymbol{Z})]$ up to three decimal places. We compare $Q_{h}\left(\boldsymbol{\theta}_{\mathbf{0}}\right)$ with the bound in (3.2), using the difference between their values as a measure of the error. The results from the simulations are shown in Table 1 below.

Table 1 Simulation results for the $\mathrm{N}(1,1)$ distribution

\begin{tabular}{l|l|l|l}
$n$ & $Q_{h}\left(\boldsymbol{\theta}_{\mathbf{0}}\right)$ & Upper bound & Error \\
\hline \hline $10^{3}$ & 0.011 & 0.457 & 0.446 \\
\hline $10^{4}$ & 0.010 & 0.145 & 0.135 \\
\hline $10^{5}$ & 0.009 & 0.046 & 0.037 \\
\hline $10^{6}$ & 0.006 & 0.014 & 0.008
\end{tabular}

We see that the bound and the error decrease as the sample size gets larger. To be more precise, when at each step we increase the sample size by a factor of ten, the value of the upper bound drops by a factor close to $\sqrt{10}$, which is expected since the order of the bound is $O\left(n^{-1 / 2}\right)$, as can be seen from (3.2).

\section{Acknowledgements}

This research occurred whilst AA was studying for a DPhil at the University of Oxford, supported by a Teaching Assistantship Bursary from the Department of Statistics, University of Oxford, and the EPSRC grant EP/K503113/1. RG acknowledges support from EPSRC grant EP/K032402/1 
and is currently supported by a Dame Kathleen Ollerenshaw Research Fellowship. We would like to thank the referee for their helpful comments and suggestions.

\section{References}

[1] Anastasiou, A. Assessing the multivariate normal approximation of the maximum likelihood estimator from high-dimensional, heterogeneous data. arXiv:1510.03679, 2015.

[2] Anastasiou, A. and Ley, C. Bounds for the asymptotic normality of the maximum likelihood estimator using the Delta method. ALEA Lat. Am. J. Probab. Math. Stat. 14 (2017), 153-171.

[3] Anastasiou, A. and Reinert, G. Bounds for the normal approximation of the maximum likelihood estimator. Bernoulli 23 (2017), pp. 191-218.

[4] Billingsley, P. Statistical Methods in Markov Chains. Ann. Math. Stat. 32 (1961), $12-40$.

[5] Cox, D. R. and Snell, E. J. A General Definition of Residuals. J. Roy. Stat. Soc. B Met. 30 (1968), 248-275.

[6] Davison A. C. Statistical Models (First ed.). Cambridge Series in Statistical and Probabilistic Mathematics, Cambridge University Press, 2008.

[7] Gaunt, R. E. Rates of Convergence in Normal Approximation Under Moment Conditions Via New Bounds on Solutions of the Stein Equation. J. Theoret. Probab. 29 (2016), 231-247.

[8] Gaunt, R. E. and Reinert, G. The rate of convergence of some asymptotically chisquare distributed statistics by Stein's method. arXiv:1603:01889, 2016.

[9] Mäkeläinen, T., Schmidt, T. K. and Styan, G. P. H. On the existence and uniqueness of the maximum likelihood estimate of a vector-valued parameter in fixed size samples. Ann. Stat. 9 (1981), 758-767.

[10] Winkelbauer, A. Moments and absolute moments of the normal distribution. arXiv:1209.4340, 2012. 